\title{
Filigrane
}

Écoutes psychanalytiques

\section{L’interprétation : pour une remise en chantier}

\section{Martin Gauthier}

Volume 27, numéro 2, 2018

La consultation psychanalytique aujourd'hui, entre héritages et remaniements

URI : https://id.erudit.org/iderudit/1055746ar

DOI : https://doi.org/10.7202/1055746ar

Aller au sommaire du numéro

\section{Éditeur(s)}

Revue Santé mentale au Québec

ISSN

1192-1412 (imprimé)

1911-4656 (numérique)

Découvrir la revue

Citer cet article

Gauthier, M. (2018). L'interprétation : pour une remise en chantier. Filigrane, 27(2), 17-27. https://doi.org/10.7202/1055746ar

\section{Résumé de l'article}

La transmission de la psychanalyse freudienne a souffert de son institutionnalisation, et des totems et tabous qui l'ont accompagnée. Comme la musique, la psychanalyse se transmet et s'interprète par le même geste, ce qui alimente la culpabilité de la trahir. L'interprétation ouvre en même temps une petite apocalypse salvatrice (Baricco) face aux pièges totémiques. L'évolution institutionnelle a contribué à appauvrir le dialogue entre la psychanalyse et la culture actuelle, tandis que cette culture s'est éloignée d'un intérêt pour le monde intérieur, dans la mouvance de ce que nous appelons une défense maniaque de la vie quotidienne, à la suite de Winnicott. L'institution psychanalytique donne des signes d'une nouvelle vitalité et les centres de consultation plus économiquement abordables font foi de ce mouvement.
Ce document est protégé par la loi sur le droit d'auteur. L'utilisation des services d'Érudit (y compris la reproduction) est assujettie à sa politique d'utilisation que vous pouvez consulter en ligne.

https://apropos.erudit.org/fr/usagers/politique-dutilisation/ 


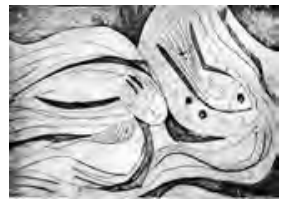

\title{
L'interprétation: pour une remise en chantier
}

\author{
Martin Gauthier
}

\begin{abstract}
Résumé: La transmission de la psychanalyse freudienne a souffert de son institutionnalisation, et des totems et tabous qui l'ont accompagnée. Comme la musique, la psychanalyse se transmet et s'interprète par le même geste, ce qui alimente la culpabilité de la trahir. L'interprétation ouvre en même temps une petite apocalypse salvatrice (Baricco) face aux pièges totémiques. L'évolution institutionnelle a contribué à appauvrir le dialogue entre la psychanalyse et la culture actuelle, tandis que cette culture s'est éloignée d'un intérêt pour le monde intérieur, dans la mouvance de ce que nous appelons une défense maniaque de la vie quotidienne, à la suite de Winnicott. L'institution psychanalytique donne des signes d'une nouvelle vitalité et les centres de consultation plus économiquement abordables font foi de ce mouvement.
\end{abstract}

Mots clés: institution; interprétation; défense maniaque; totem; tabou.

\begin{abstract}
The transmission of Freudian psychoanalysis has suffered from its institutionalisation and the totems and taboos which accompanied it. Like music, psychoanalysis is transmitted and interpreted by the same action, which feeds the guilt involved in betraying it. At the same time the interpretation opens a small saving apocalypse (Baricco) in face of the totemic traps. The institutional evolution has contributed to impoverishing the dialogue between psychoanalysis and today's culture, while this culture has moved away from any interest in the inner world through what we, following Winnicott, call a manic defence of everyday life. The psychoanalytical institution is giving signs of new vitality and the more economically affordable centres of consultation are evidence of this movement.
\end{abstract}

Keywords: institution; interpretation; manic defence; totem; taboo.

a ville de Québec fêtait en 2008 ses 400 ans de fondation, Montréal célèbre en 2017 son $375^{\circ}$ anniversaire tandis que la Société canadienne de psychanalyse souffle ses soixante bougies ${ }^{1}$. Que retenons-nous de ces fondations? En 1608, des vingt-huit personnes installées à Québec pour le premier hiver, seules huit survécurent. Le fondateur de la ville, brillant cartographe et patient architecte de la Nouvelle-France, Samuel de Champlain, garde aujourd'hui un visage inconnu (on lui a affublé une tête imaginaire, inspirée d'un certain Michel Particelli, voleur de profession), une date de naissance incertaine et une tombe encore introuvable. Lorsque nous évoquons notre 
histoire et notre héritage, de quel fondateur, de quelle fondation nous réclamons-nous? Héritiers de l'hiver et porteurs des traces laissées jusqu'à nous, constatons que nos mémoires sont sélectives: nous nous souvenons de certains vivants en les déguisant selon nos désirs et nos tourments, tandis qu’il y a tant de morts oubliés. Avec les survivances, nous fondons nos pratiques actuelles au gré des nouvelles saisons.

Sigmund Freud a été un autre grand explorateur et cartographe. Alors qu'il se sentait vieillir et qu'il observait les conflits que sa succession agitait, il se servit de tiers, les peuples primitifs et les enfants, à la manière d'un écran extérieur, pour écrire son grand texte sur l'ambivalence, Totem et tabou (1913). La réflexivité que cet écran tiers lui permettait reprenait celle déjà inscrite dans le langage, ouvrant la distance nécessaire au déploiement de sa pensée. Les prédécesseurs nous servent de même manière à penser le présent. La stratégie de Freud était alors d'autant plus intéressante que son propos concernait l'âme et le devenir de ce qui est mangé. Le père de la psychanalyse enracina la filiation dans le terreau de l'ambivalence et reconnut en celle-ci une propriété de tout rapport humain, en particulier dans la dimension fondatrice qu'il appelle l'identification. Il voulut par la suite clarifier ce que comporte cette construction identificatoire ${ }^{2}$ qui resta toujours à ses yeux fille de l'ambivalence. Avec le jugement d'appropriation et le moi-plaisir purifié introduits par Freud dans La négation (1925), il pouvait donner une impression d'un simple choix dichotomique entre dedans et dehors, entre bon et mauvais; mais, dès Totem et tabou, un double héritage interne était reconnu: toute internalisation a un destin duel sous forme d'un totem et d'un tabou.

Freud annonçait déjà sa division des instances moïque et surmoïque. Il soulignait l'ambivalence du processus d'appropriation et l'inévitable division interne issue du processus. Le repas totémique ne saurait être célébré sans devoir et sans idéal. Cela soulevait déjà une question cruciale: qu'est-ce qui détermine le devenir de l'objet une fois «mangé»? Qu'est-ce qui différencie les «identifications» qui constituent le moi de celles qui donnent forme au surmoi? La question se posera particulièrement au sein du complexe d'Edipe et elle traversera Le moi et le ça (1923) sans obtenir une réponse nette (Gauthier, 2013). Jusque dans son Moïse testamentaire, Freud s'intéressera au devenir intérieur et collectif de ce qui est tué et mangé, aux totems et aux tabous alors érigés.

L'histoire des institutions psychanalytiques permet de constater combien la filiation est affaire d'ambivalence, d'amour et de haine cannibaliques 
et castrateurs, de meurtre et de culpabilité. La vie institutionnelle n'estelle pas jalonnée de conflits passionnels qui surgissent tout à coup, nous prennent toujours à découvert et marquent l'évolution du groupe? Et ces combats se livrent si souvent sur le terrain de la transmission et de la filiation. Le groupe avance en cherchant à élaborer ce qui reste seulement agi, ce qui est une manière de souligner la nécessité de penser les origines toujours en mouvement, en re-fondation.

Une idée en devenir voudra s'organiser pour favoriser son développement. Mais une fois cette idée instituée, une fois inscrite dans une institution, avec pignon sur rue et règles de déploiement, sa vitalité s'étiole et se fige aisément au profit des mots d'ordre qui paradoxalement voudraient garantir sa pérennité. Les craintes de perdre la découverte initiale l'érigent en système et entraînent une rigidité grandissante qui étouffe l'élan fondateur. La découverte psychanalytique avait permis d'appréhender la réalité sous un nouveau jour. Elle ouvrait une perspective qui repoussait les frontières. Elle faisait subversion face aux règles d'usage et portait l'espoir d'une nouvelle liberté. Le tranchant de l'idée s'émoussa quand elle cessa d'être en chantier sous le poids des acquis à préserver. Bob Dylan ne dit pas moins en chantant: "That he not busy being born is busy dying ${ }^{3}{ }$. Quelque part en route, la réponse acquise a tronqué le dialogue.

\section{L'interprétation: une petite apocalypse salvatrice}

L'interprétation constitue une petite apocalypse salvatrice devant ce problème involutif. La formule est d'Alessandro Baricco (1992), musicologue et romancier. Il a écrit un bel essai sur la transmission de la musique qu'il a intitulé L'âme de Hegel et les vaches du Wisconsin. Son étude porte sur la musique classique et la musique contemporaine, sur leur pertinence actuelle et leur place dans notre monde: «Est-ce que ça a un sens ou est-ce que c'est seulement un exercice gratuit, destiné à quelques élus qui ont dressé leur tente à l'extérieur du monde?»(Baricco, 1992, p. 10). Cette interrogation touche au vif de celle du psychanalyste qui questionne l'évolution de sa propre discipline et sa place dans la culture actuelle.

Baricco souligne une caractéristique particulière de la musique: elle se transmet et s'interprète par un même et unique geste. Et ce geste qui la conserve et la transmet vient aussi la corrompre du fait des variables de jeu infinies. Ne dirions-nous pas la même chose pour la psychanalyse, qui se transmet et s'interprète elle aussi dans un même mouvement? Il y a maintenant certes une vaste littérature et un docte savoir psychanalytiques 
s'étendant jusqu'à un enseignement universitaire. Néanmoins tout l'art d'être psychanalyste, fondé sur l'expérience du transfert et du contre-transfert (des deux côtés du divan pour ceux devenus psychanalystes eux-mêmes), ne s'acquiert que dans le geste lui-même, comme dans le cas de la musique. Jean-Luc Donnet (1995) abordait cet enjeu en décrivant l'écart théorico-pratique, où il repère une prescription éthique pour laisser les coudées franches au champ de la pratique. Seule l'interprétation insuffle son mouvement à un savoir qui autrement peine à s'incarner et fausse l'expérience.

Toutefois, comme le souligne Baricco pour le musicien, une telle situation n'est pas sans conséquence, car elle condamne à un complexe éternel de culpabilité: la crainte constante de trahir l'original, avec la possibilité de le perdre, de l'endommager ou le corrompre à jamais. Cette trahison accompagne les pas de l'interprète à qui est confiée la suite de la précieuse psychanalyse. L'interprétation est une petite apocalypse salvatrice mais ce salut se gagne sur la culpabilité de trahir l'idéal. Interpréter tisse ensemble mort et salut pour que respire le présent.

Quelle est-elle donc cette authentique psychanalyse qu'il faudrait préserver? Du vivant du fondateur et surtout après sa mort, particulièrement sous la plume d'Ernest Jones, un mythe du héros a été constitué autour de la figure de Freud. Ce mouvement hagiographique trouve sa réciproque dans les attaques contre la personne de Freud et les travers de sa vie personnelle, les deux volets voulant concentrer et figer le pouvoir de l'idée à la figure du fondateur. L'institution psychanalytique, née dans l'agitation des conflits contemporains à Totem et tabou, s'est développée autour d'une psychanalyse idéale à sauvegarder d'une barbare corruption. La générativité des idées nouvelles et leur capacité à dialoguer avec la vie collective ont décliné à mesure que l'idéalisation a pris le haut du pavé. Cette observation ne signifie pas l'absence de toute évolution originale ultérieurement, mais force est de constater combien l'idéalisation freine et combien la vitalité du chantier des premières décennies s'est affaiblie pendant que l'institution gagnait en prestige social.

«L'Histoire est une prison aux barreaux fragiles. Et on continue à monter la garde autour d'un prisonnier évadé depuis longtemps», écrit Baricco (1992, p. 47) pour dénoncer l'aporie de l'idéalisation des origines. Qui est donc ce prisonnier que l'on croit illusoirement garder, plutôt que de suivre ses traces dans les nouvelles saisons? Nous nous attachons aux figures des fondateurs, à nos pères, mères et maîtres, comme s'ils détenaient la vérité de la psychanalyse. Nous nous saisissons d'eux, sous forme de totems et de 
tabous, et nous en sommes simultanément saisis, condamnés à monter la garde pour ne pas les perdre. Les psychanalystes se transmettent des copies qui sont autant d'originaux, mais au-dessus desquelles flotte l'ombre d'un étalon qui s'est enfui. Pire, ce dernier n'a jamais existé. La pratique de Freud ne correspond pas à l'idéal recherché et la manière de mener une analyse a évolué avec les générations d'analystes et avec la pratique de chacun. Il y a plusieurs «vrais» Freud qui sont autant d'interprétations de l'œuvre du fondateur. Il y a eu une suite de lectures significatives; celle de Ferenczi, de Klein, de Winnicott, de Bion, de générations de psychanalystes jusqu'à nous. Il y a eu des psychanalystes en quête de vérité. Celle-là est notre exigence. Mais comme il est facile d'y substituer un autre impératif, sévère, idéalisé, garant de la psychanalyse «vraie de vraie»!

La psychanalyse freudienne a tôt fait le choix de développer ses propres institutions, autonomes des pouvoirs universitaires, corporatifs ou étatiques, et ses propres canaux de communication (congrès, revues). Être psychanalyste devint vite l'identité valorisée de ses praticiens venus de champs professionnels divers. Même quand le statut médical garantissait le sérieux et la valeur sociale, notamment aux États-Unis qui insistèrent longtemps pour que seuls les médecins puissent voir leur formation psychanalytique reconnue, c'est le titre de psychanalyste qui accordait l'identité recherchée, avec le prestige supplémentaire d'appartenir à un groupe sélect. Nous sommes là du côté du totem, d'une définition identificatoire qui ne vient jamais sans tabous. Ceux-ci se firent plus exigeants et sévères à mesure que le totem gagna en valeur symbolique et que ses membres purent se sentir culturellement et moralement privilégiés.

J'esquisse à trop grands traits une évolution qui participa à l'isolement social croissant des institutions psychanalytiques, campées dans leur devoir de préserver la psychanalyse contre toute corruption externe, soucieuses d'un statu quo conservateur, valorisant une pratique à l'abri du monde ambiant et jalouse de son extraterritorialité. Pendant ce temps, la société évoluait, développait de nouveaux langages, ouvrait des territoires inédits. Les psychanalystes ont sans doute trop longtemps négligé l'ampleur des changements sociaux, d'autant qu'ils continuaient à profiter des avantages économiques de leur réputation antérieure. Aujourd'hui, pour survivre et croître, la psychanalyse doit redevenir une idée en devenir, articulée à la culture actuelle. Il lui faut retrouver sa vitalité en réinventant son dialogue avec le temps présent. 


\section{L'institution psychanalytique aujourd'hui}

L'Association psychanalytique internationale est aujourd'hui une organisation qui compte près de 13000 membres et plus de 5000 candidats répartis dans soixante-sept pays et deux mille villes. Elle est divisée en trois grandes régions officielles, à savoir l'Europe, l'Amérique du Nord et l'Amérique du Sud. L'Asie compte déjà quelques Sociétés locales mais ne forme pas encore une région officielle. C'est pourtant là qu'a lieu actuellement la croissance la plus remarquable quant à l'intérêt pour la psychanalyse et pour la formation psychanalytique. L'Europe de l'Est et l'Amérique du Sud démontrent aussi un essor significatif. Le portrait international qui se dessine met en relief le rôle du développement socio-économique et politique: le passage récent à des gouvernements plus démocratiques et à des économies de marché favorise la croissance de la psychanalyse, dans un contexte de liberté accrue et d'intérêt grandissant pour le développement personnel.

Ces statistiques sont impressionnantes et peuvent donner à penser que la psychanalyse a le vent dans les voiles. Nous savons combien ce n'est pas le cas en Occident, particulièrement dans les pays industrialisés où elle est implantée depuis longtemps. Outre le désillusionnement que la psychanalyse suscita par un espoir démesuré et son usage sans discrimination suffisante des fonctionnements mentaux, outre sa trop grande arrogance face à d'autres avenues thérapeutiques, elle se heurta à des critiques sévères quant à son coût et son manque de preuves quantitatives d'efficacité. Les impératifs budgétaires l'ont maintenant mise à l'index pour favoriser des approches à court terme qui permettent une gestion plus serrée des demandes de soins. Parallèlement, la pharmacologie a offert une alternative aux interventions psychothérapiques et favorisé un désintérêt de la vie intérieure et relationnelle au profit d'une régulation affective par des agents chimiques externes. L'usage des drogues récréatives propre auparavant aux sous-cultures en marge du pouvoir dominant a progressivement été récupéré sous une forme commerciale au sein du courant dominant pour devenir une façon privilégiée de moduler les états mentaux et d'éloigner la souffrance psychique.

À l'heure actuelle, les institutions psychanalytiques ont donné un coup de barre et cherchent à corriger la situation. La réalité est abordée avec un nouveau réalisme. C'est notamment le cas au niveau de l'administration de l'Association psychanalytique internationale où des changements significatifs, bloqués auparavant, sont enfin devenus possibles. Il y a une dizaine d'années, différents modèles de formation ont été officiellement reconnus alors que jusque-là l'institution s'entêtait à prétendre que le modèle élaboré 
par Max Eitingon à Berlin au début des années 1920 était le seul valable Aujourd'hui ce modèle Eitingon est lui-même l'objet de révisions et une saine discussion a lieu pour rendre la formation psychanalytique et la pratique quotidienne plus aptes à dialoguer avec la culture actuelle. Parallèlement, des initiatives de rayonnement et de sensibilisation sont apparues dans plusieurs villes, signe d'un désir de sortir hors du cadre traditionnel. La modélisation théorique du travail témoigne aussi d'une ouverture au changement. L'activité du psychanalyste se redéfinit, autant dans la séance que dans le champ social, en se dégageant de la hantise de la suggestion. La réalité clinique des troubles précoces de mentalisation et de symbolisation entraîne une révision des pratiques pour favoriser la construction de l'espace psychique personnel.

\section{Une tendance culturelle: la défense maniaque de la vie quotidienne}

Il ne s'agit pas de condamner les institutions de façon aveugle. Elles sont non seulement nécessaires mais constitutives de notre vie sociale. C'est aussi ce que Freud fit valoir par son mythe de la horde originaire, dans la foulée de Atkinson et Darwin. En soulignant la dévitalisation de l'idée première au sein de l'institution, je schématise un enjeu parmi un ensemble qui déterminera si l'institution demeure davantage un vrai groupe de travail, en contact avec la réalité extérieure, plutôt qu'un groupe qui s'en isole de plus en plus. En utilisant la théorisation de Wilfred Bion (1961) issue de son expérience pendant la Deuxième Guerre mondiale, il est possible d'opposer le groupe de travail au groupe fonctionnant avec une hypothèse de base fantasmatique (dépendance, attaque-fuite, couplage) qui a pris le dessus sur une véritable prise en compte de la réalité. Sous l'emprise des hypothèses de base, le groupe psychanalytique cessait de travailler sa rencontre avec la réalité sociale.

À l'exemple de la théorisation de Bion, la psychanalyse offre des outils pour réfléchir à la culture occidentale actuelle. Il n'est pas aisé de saisir les mouvements de fond qui insidieusement donnent forme et direction à la culture. Là aussi, comme pour l'individu, la traduction des événements vient toujours après coup. Notre déficience à pouvoir appréhender l'ensemble de la réalité limite notre entendement. Si mon propos vise d'abord la psychanalyse, il nous faut constater que la décroissance observée ne concerne pas qu'elle seule, mais l'ensemble du champ de la psychothérapie. La psychanalyse a été une chef de file, première championne de la pertinence du travail 
psychothérapeutique pour l'individu et pour la collectivité, et son déclin met en relief une tendance plus générale: celle d'un désintérêt croissant pour le monde intérieur et pour le travail d'introspection. Comment expliquer cette évolution qui survient après des années où, au contraire, le développement personnel avait bonne presse, dans la foulée des changements sociaux des années 60 ? On voudra mettre des bémols à cette affirmation générale et évoquer par exemple l'intérêt que suscitent actuellement les démarches méditatives, notamment celles de "pleine conscience». Peut-on penser que ces nouvelles mouvances cherchent justement à réagir à la tendance opposée, celle où l'individu cherche son équilibre à l'extérieur de soi? De plus, elles tiennent à se distinguer d'une psychothérapie, profitant du courant social de méfiance face à l'exploration du monde intérieur.

Une autre notion issue de la psychanalyse peut éclairer cet aspect de la culture occidentale actuelle dont je fais l'hypothèse. En 1935, Winnicott exposait à la Société britannique ses idées sur la défense maniaque, que Klein (1934) avait décrite peu auparavant. Il y insistait sur la différence entre la réalité intérieure (ou psychique) et la fantasmatisation. Tout son propos semble tirer sa source d'une expérience accrue, d'une connexion plus intime, de ce que sous-tend la notion de réalité intérieure. Cette nouvelle prise en compte a été gagnée sur les forces qui s’y opposent: «Cela fait partie de sa propre défense maniaque que d'être incapable de donner sa pleine signification à la réalité intérieure ( Winnicott, 1935, p. 15). Le respect de notre réalité psychique, ajoutait-il, dépend de l'angoisse dépressive qui nous habite.

Winnicott propose que nous lisions la réalité extérieure à travers le prisme des fantasmes, toujours omnipotents, que nous élaborons pour fuir l'impact de la réalité intérieure. À la défense maniaque pathologique, caractérisée par la manipulation omnipotente, le contrôle et la dépréciation méprisante, il oppose une variante bénigne, une défense maniaque de la vie quotidienne. Il donne l'exemple du music hall, du poste de radio toujours ouvert, de la ville au bruit incessant et aux lumières jamais éteintes, ou encore des carnets mondains des journaux. Une réassurance face au monde intérieur est ainsi offerte par l'intermédiaire de la réalité externe. La dimension maniaque y sera difficilement observable par l'individu compte tenu de la nature même de la défense, qui stimule et éloigne du monde intérieur (et des considérations «sérieuses»). L'introspection n’y est pas favorisée, contrairement à ce qui se passe lors d'un mouvement dépressif.

La défense maniaque de la vie quotidienne que décrit Winnicott semble annoncer une tendance de l'évolution culturelle de l'Occident depuis la fin 
de la Deuxième Guerre mondiale 5 . Devant l'accélération et la complexification de la vie urbaine, à la faveur des avancées techniques et technologiques, un rôle accru est confié à la consommation, notamment celle des vastes produits de l'industrie du divertissement, multipliés par l'ouverture du champ virtuel. La nouvelle frontière n'est plus celle de territoires insoupçonnés à l'intérieur de soi mais celle du nouveau continent virtuel avec ses choix qui ne cessent de croître. La société marchande a remplacé les rituels religieux par une quête matérielle. L'individu est invité à trouver refuge du côté de l'excitation extérieure face à une vie intérieure désinvestie. Même le champ des neurosciences est généralement abordé à la manière d'une machine, réduisant facilement l'agence subjective à des déterminismes physicochimiques externes, selon le présupposé physicaliste. Là comme ailleurs, le médium a déclassé le message.

La psychanalyse, comme toutes les psychothérapies qui invitent à se tourner vers le monde plus trouble de la subjectivité, nage à contre-courant de la direction qu'emprunte la société marchande, soutenue par et soutenant la défense maniaque de la vie quotidienne. C'est une dimension qu'il faut sérieusement considérer dans le nouveau dialogue que souhaitent engager les psychanalystes.

\section{En conclusion: des lieux de dialogue}

Comment la psychanalyse rencontre-t-elle le temps présent? Comment reste-t-elle une idée vivante et une pratique signifiante dans la culture actuelle? J'ai voulu souligner le rôle de la dimension institutionnelle, inscrite dans le champ social et les courants qui le façonnent. Des totems et des tabous se dressent toujours dans l'évolution culturelle et institutionnelle, individuellement et collectivement.

La dimension sociale et institutionnelle se prolonge jusque dans la consultation thérapeutique, sous l'abri de l'intimité des cabinets. Elle pèse notamment dans ce que Chiantaretto appelle l'interlocution interne de l'analyste en séance ${ }^{6}$. Celui-ci s'est intéressé au cadre interne de l'analyste et sa mise en tension dans la séance. Cette dimension inscrit le praticien au sein d'une filiation, notamment par son analyse personnelle. Elle le confronte en particulier à ce qui demeure inanalysé chez lui et qui renvoie à l'inanalysé de son propre analyste, dans une transmission blanche de l'inanalysé d'une génération à la suivante. Le dialogue Freud-Ferenczi offre un exemple paradigmatique du malentendu. Cet inanalysé peut faire barrage mais il est aussi l'aiguillon qui pousse vers l'avant. Il est un moteur de la petite apocalypse 
salvatrice que représente l'interprétation. L'inachevé ouvre aux nouvelles pistes interprétatives et garde au chantier sa vitalité. Au contraire, c'est l'illusoire achèvement que l'idéalisation promeut qui isole tant l'individu que l'institution.

Un enjeu actuel est certainement celui de rapprocher la psychanalyse de ceux et celles qui peuvent s'y intéresser et en profiter. La rendre abordable, de part et d'autre. Cela implique une proximité de lieu et de temps, un coût accessible et un langage partageable. Les lieux de consultation psychanalytique offrant des traitements à des prix qui tiennent compte des capacités de payer constituent aussi des carrefours pour y rencontrer les nouvelles formes que prend la souffrance, individuelle et sociale, comme celle aujourd'hui associée au choix du genre et à l'automutilation. De telles initiatives germent et se développent malgré les défis économiques ${ }^{7}$. Elles incarnent un désir de rencontre, un exemple du souffle qui permet d'avoir espoir en un nouveau dialogue entre la psychanalyse et le monde actuel.

\author{
Martin Gauthier \\ martin.gauthier3@sympatico.ca
}

\title{
Notes
}

1. Avec le parrainage de la Société britannique de psychanalyse, la Société canadienne de psychanalyse est officiellement reconnue par l'Association psychanalytique internationale (API) le 31 juillet 1957, lors du 20e Congrès de l'API à Paris. Elle devenait une société composante de la grande association créée par Freud et les premiers fondateurs en 1910. Dans le grand courant social qui sera baptisé la Révolution tranquille, la psychanalyse canadienne débutera à Montréal et se déploiera ensuite vers l'Ouest, reprenant le parcours de la colonisation européenne de ce qui est devenu la confédération canadienne.

2. Pensons aux grands écrits ultérieurs: Deuil et mélancolie (1915), Psychologie des foules et analyse du moi (1921) et Le moi et le ça (1923).

3. "Celui qui n'est pas occupé à naître est occupé à mourir» (Bob Dylan, It's alright, Ma (I'm only bleeding)).

4. Le modèle de formation élaboré par Max Eitingon et l'Institut de formation de Berlin au début des années 1920 comporte trois axes, soit l'analyse personnelle, les cures supervisées et les séminaires cliniques et théoriques. Des critères stricts (standards minimaux) balisent la reconnaissance du cursus de formation. Ce modèle a fait l'objet de différentes critiques (lire notamment Kernberg, 2002), mais demeure celui dominant internationalement.

5. Je m'aventure ici du côté d'une interprétation sociologique, loin de mes repères habituels, ce que d'aucuns trouveront sans doute téméraire ou, pire, déplacé. Comme dans un texte antérieur (Gauthier, 2014), mon souhait est d'unir une perspective psychanalytique à une réflexion commune sur notre paysage actuel, espérant que les diverses perspectives puissent enrichir notre vision toujours trop partielle. 
6. Voir notamment l'article de J.-F. Chiantaretto, «Cadre interne, transfert et contre-transfert» dans le présent recueil.

7. Le colloque d'octobre 2016 rassemblait des représentants de diverses cliniques et centres de consultation poursuivant ce désir d'articuler la clinique psychanalytique à des clientèles autres que celles qui frappent à la porte des cabinets privés des psychanalystes.

\section{Références}

Baricco, A. (1992). L'âme de Hegel et les vaches du Wisconsin. Paris: Albin Michel, 1998.

Bion, W. R. (1961). Recherches sur les petits groupes. Paris: Presses universitaires de France, 1965.

Donnet, J.-L. (1995). Le divan bien tempéré. Paris: Presses universitaires de France.

Freud, S. (1913). Totem et tabou. Paris: Petite Bibliothèque Payot, 1980.

Gauthier, M. (2014). La résistance de la nature humaine. Filigrane, 23(1), 12-25.

Gauthier, M. (2013). Les origines meurtrières du surmoi. Revue française de psychanalyse, 77(5), 1488-1494.

Kernberg, O. (2002). La formation psychanalytique: quelques préoccupations. Revue française de psychanalyse, 66, 227-251.

Klein, M. (1934). Contribution à l'étude de la psychogenèse des états maniaco-dépressifs. Dans auteur (1967), Essais de psychanalyse. Paris: Payot.

Winnicott, D. W. (1935). La défense maniaque. Dans auteur (1969), De la pédiatrie à la psychanalyse (p. 15-32). Paris: Payot. 